\title{
Evaluation of mir-377-3p Expression in Patients with Multiple Sclerosis
}

\author{
Majid Amini Khorasgani ${ }^{a^{*}}$, Parisa Mohammady Nejad ${ }^{a}$, \\ Mohammad Mehdi Moghani Bashi ${ }^{\mathrm{b}}$, Mohammad Hedayati ${ }^{\mathrm{a}}$ \\ ${ }^{a}$ Department of Genetics, Faculty of Basic Sciences, Islamic Azad University, Shahrekord Branch, Shahrekord, Iran. \\ ${ }^{b}$ Department of Genetics, Faculty of Basic Sciences, Islamic Azad University, Kazerun Branch, Kazerun, Iran.
}

Received 04 March 2019; Accepted 21 May 2019

\begin{abstract}
One of the most recent studies in the field of genetics is to investigate the role of microRNAs as biomarkers for the diagnosis of multiple sclerosis. It is an inflammatory and degenerative disease of the central nervous myelin, which is manifested in numerous small and large plaques in the white matter of the brain and spinal cord. Formerly called has-miR-377-3p, miR$377-3 p$ is located within the chromosomal region 32q14, and is located in the SOD gene. SOD (Superoxide Dismutase) is a gene located on chromosomal region 22q21 and the protein encoded by this gene is the superoxide dismutase enzyme. The aim of this study was to evaluate the expression of miR-377 in people with RRMS (Relapsing-Remitting Multiple Sclerosis) and healthy individuals in the Isfahan population. The study included 49 patients with RRMS and 52 healthy individuals who had no history of autoimmune and inflammatory disease. Total RNA was extracted from the blood lymphocytes of the study subjects using Ficol and Trizol and then made using miRNA cDNA, cDNA specific kit, and expression was measured by real-time RT PCR in healthy subjects and patients. Was. According to the results, miR-377-3p expression was higher in patients than in healthy subjects $(\mathrm{P}=0.036)$ and the sensitivity and diagnostic value of miRNA was AUC $=0.80$ (Area under the Curve). ) is. The results were consistent with previous studies and miR-377-3p could be used as a biomarker for the diagnosis of Multiple Sclerosis.
\end{abstract}

Keywords: Multiple Sclerosis; RRMS; miR-377-3p; SOD Gene.

\section{Introduction}

Multiple sclerosis is an inflammatory disease of the central nervous system that is associated with the inflammation and disappearance of the nerve sheaths of the body called myelin; it is a sheath that surrounds nerve fibers and plays an important role in the rapid transmission of nerve waves. Normally, though, the sheaths of the nerve are transmitted quickly and allow the body to perform harmonious movements. Symptoms vary depending on demineralization sites and missing axons, including impaired vision, coordination, balance and movement [1]. The disease is usually present in young people and is more common in women than men; more than two million people worldwide have the disease [2]. There are three major processes involved in the pathogenesis of the disease: genes, inflammation, and neuronal destruction. The most common age for MS is between 20-40 years. Prevalence of MS in infancy is also low after age 50[3]. The prevalence of MS in Tehran in 2009 was 51.9 and in Isfahan in 2010, 73.3 cases per 100,000,100 were reported. MS is the second leading cause of youth disability in Iran, with an increase in the number of these patients per year $(7,7)$ [4]. Although the exact factors that cause MS are not known, the following factors may contribute to the development of MS, including DNA and mitochondrial dysfunction, viral infections, and hypoxia), Oxidative

* Corresponding author: amini_microbiology2011@yahoo.com

http://dx.doi.org/10.28991/SciMedJ-2019-0102-2

> This is an open access article under the CC-BY license (https://creativecommons.org/licenses/by/4.0/).

(C) Authors retain all copyrights. 
stress, lack of light or vitamin D levels, and increased macrophages (microglia) and lymphocytes in the brain [5].

miR-377-3p, formerly called has-miR-377-3p, is located within the chromosomal region 32q14, which has been reported in kidney diseases such as diabetes and is located in the SOD gene [6]. (Table 1).

Table 1. Reaction between miR-377-3p and the SOD gene in the microrna base

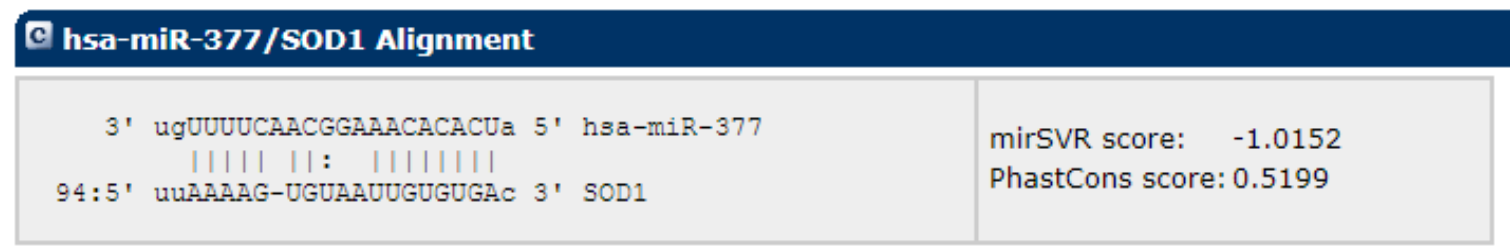

Mouseover a miRNA mature name to see the miRNA/SOD1 alignment.

SOD (Superoxide Dismutase) is a gene located on chromosomal region 22q21 and the protein encoded by this gene is the superoxide dismutase enzyme that is present in humans in three forms: SOD1, SOD2 and SOD3 [7]. Superoxide dismutase 1 or SOD1 is a cytosolic enzyme in humans that is encoded by the SOD gene with International Code 6647. It is a potent antioxidant that protects the body from the damage caused by superoxide, a toxic free radical produced in the mitochondria by converting superoxide radicals to oxygen and hydrogen peroxide. Free radicals produced during cell metabolism can accumulate and damage mitochondrial DNA, nuclei and proteins within the cell. Superoxide is one of the most abundant reactive oxygen species that can damage cellular macromolecules. It is produced in mitochondria and is believed to be involved in cellular signaling pathways. Under normal conditions, the mitochondrial electron transport chain is the major source of superoxide production, which converts more than $5 \%$ of molecular oxygen to superoxide, but increases in pathological conditions. Superoxide dismutase (SOD) is a family of enzymes that play a major role in the conversion of superoxide to $\mathrm{H} 2 \mathrm{O} 2$; free radicals are also produced during cellular metabolism such as respiratory burst and enzymatic reactions [6].

Cells usually have defense mechanisms against ROS-induced damage, and this is a serious issue when ROS production exceeds its antioxidant capacity or the cell's antioxidant system is damaged and this lack the balance between ROS cellular production and the cell's inability to defend itself is called oxidative stress. Oxidative stress causes cell damage and ultimately cell death. ROS is mainly produced in the brain and nerve tissues by the metabolism of neurotransmitters such as glutamate and are the major source of ROS in the CNS. In addition, metals such as iron that mediate ROS production reactions are present in some areas of the brain at high concentrations. Other sources of ROS in the CNS include the continued use of oxygen in the mitochondria, enzymatic pathways such as xanthine dehydrogenase, lipoxygenase, cyclooxygenase and NADPH oxidase, as well as non-enzymatic mechanisms such as catecholamine self-oxidation. ROS Attack Glia and Neurons These cells no longer carry mitosis division, so they are highly sensitive to ROS-mediated damage and lead to nerve damage. Inflammation also increases the level of ROS and leads to oxidative stress. One of the most abundant sources of ROS other than the mitochondrial electron transport chain is the activated microglial respiratory burst system, which induces nitric oxide synthase cytokines and oxygen and nitrogen free radicals leading to myelination and axonal damage. In MS and EAE. Studies in MS patients have shown that free radicals in the CNS of people with MS are more active than normal people, and they also have deficiencies in antioxidant enzymes. ROS also damages mitochondrial DNA and results in a decrease in oxidative phosphorylation and thereby loss of neurons [8].

The need for preventive or timely treatment in relation to this complex disease is not covered by anyone and in addition many of them have harmful side effects which are difficult for patients to tolerate. Over the past two decades, with the introduction of microwave RNAs as regulators of gene expression at the post-transcriptional level, another level of complexity in gene expression regulation has emerged. However, microRNAs have been able, with their potential, to give great promise to genetic researchers in the treatment of complex diseases such as cancer and autoimmune diseases such as MS. Research has shown that these small RNA molecules can provide a therapeutic pathway for disease as well as biomarkers for evaluating the therapeutic efforts and progression of the disease [9]. One of the important steps in the development of the nervous system is the formation of synapses between nerve cells and the expression of specific proteins in the synapse to enhance neuronal communication. According to available information, some microRNAs are specifically expressed in dendrites and play important roles in neuronal and synaptic plasticity [10, 11].

Schwann cells in the peripheral nervous system and oligodendrocyte cells in the central nervous system play a key role in the formation of the myelin sheath and thus play an important role in the normal function of the nerves. The presence of myelin in the myelinated axons leads to a rebound conduction and, consequently, to the nerve conduction rapidly. Recent studies have reported an important role for microRNAs in Schwann and oligodendrocyte proliferation, differentiation and myelination [12, 13]. Mice lacking the Dicer1 enzyme, a key enzyme in microRNA production, are severely defective in myelination, indicating the role of microRNAs in the differentiation of Schwann and oligodendrocyte cells. Although Schwann cells in the peripheral nerves and oligodendrocytes in the central nerves are 
responsible for myelinization, some aspects of the expression and regulation of microRNAs in these two types of cells are similar. This suggests that similar microRNAs may be involved in the differentiation of myelinating cells $[12,14]$. Several microRNAs including miR-138, miR-219, miR-228 and miR-17-92 microRNAs are highly expressed in oligodendrocytic precursors and target specific genes.

\section{Materials and Methods}

This study is an analytical-observational study. Approved and predicted target genes were sequenced using the database (http://www.microrna.org), respectively, and then 49 patient samples with RRMS using lumbar puncture methods. And MRI were identified as well as 52 healthy individuals without a history of disease from the MS clinic of Alzahra Hospital in Isfahan, approved by a neurologist and completed a questionnaire and informed consent form (Table 2).

Table 2. Clinical information of patients and healthy individuals.

\begin{tabular}{ccc}
\hline Specification & MS patients & Healthy people \\
\hline number of samples & 49 & 52 \\
Gender (number) & 28 women 21 men & 28 women 24 men \\
Average age (years) & $9 / 10 \pm 37 / 02$ & $10 / 57 \pm 33 / 86$ \\
\hline Average incidence age & & $8 / 40 \pm 30 / 64$ \\
\hline
\end{tabular}

\subsection{RNA Extraction and cDNA Synthesis}

Blood samples were collected from each individual in $3 \mathrm{ml}$ tubes containing ethylenediaminetetraacetic acid and then extracted with PBMCs using Biosera France's Ficol and then total RNA was extracted with Trizol from SigmaAldrich USA and then quantified. RNA was analyzed by quantitative ultraviolet absorption spectroscopy with concentration and purity of extracted RNA samples. The concentration of RNA extracted from the samples was measured in $\mathrm{ng} / \mu \mathrm{l}$ and the absorbance ratio of 240/280 was measured using Thermo $2000 \mathrm{C}$ Nano Drop of Thermo American. 2 microliters of this RNA was transformed into cDNA using Exiqon® miRCURY LNA TM Universal RT microRNA PCR product from Denmark (Exiqon).

\subsection{Internal Control and Desired miRNA}

U6 housekeeping gene, commonly used in miRNA expression studies, was selected as an internal control. Its expression stability and normalization were evaluated. All primer sequences are from miRanda site and designed by Exiqon Denmark.

\subsection{Real Time RT PCR}

The RT PCR reaction was performed using SYBR Green Kit, a unique kit equipped with $10 \mu 1$ reaction. The final concentration of each of the sweep primers and the U6 gene was $0.3 \mu \mathrm{M}$. Fluorescence was measured by ABI step one of ABI USA and data was analyzed by SPSS software.

\subsection{Data Analysis}

Raw data were analyzed by Real Time RT PCR and amplification efficiency and CT numbers were obtained for each reaction. CT (cycle threshold) refers to the number of cycles required to pass the fluorescent signal beyond the background or threshold signal level. The level of CT is inversely proportional to the target nucleic acid in the sample, meaning that the lower the level of CT, the higher the target nucleic acid in the sample, and vice versa. Then, the gene expression changes were studied using SPSS software and T-test. The significance level of P $<0.05$ was considered and then the expression level of the genes was quantified using miR-2 data. Balanced and interpreted.

\section{Results}

According to the results, miR-377-3p expression in patients was higher than healthy individuals $(\mathrm{P}=0.036)$ and sensitivity and diagnostic value of miRNA was AUC $=0.80$ (Area under the Curve).

\subsection{Findings}

Mean and standard deviation of miR-377-3p expression were measured in healthy and patient groups. Healthier (Figure 1). The sensitivity and the diagnostic value of this miRNA in ROC Curve analysis were equal to AUC $=0.80$ (Area under the Curve) (Figure 2). 


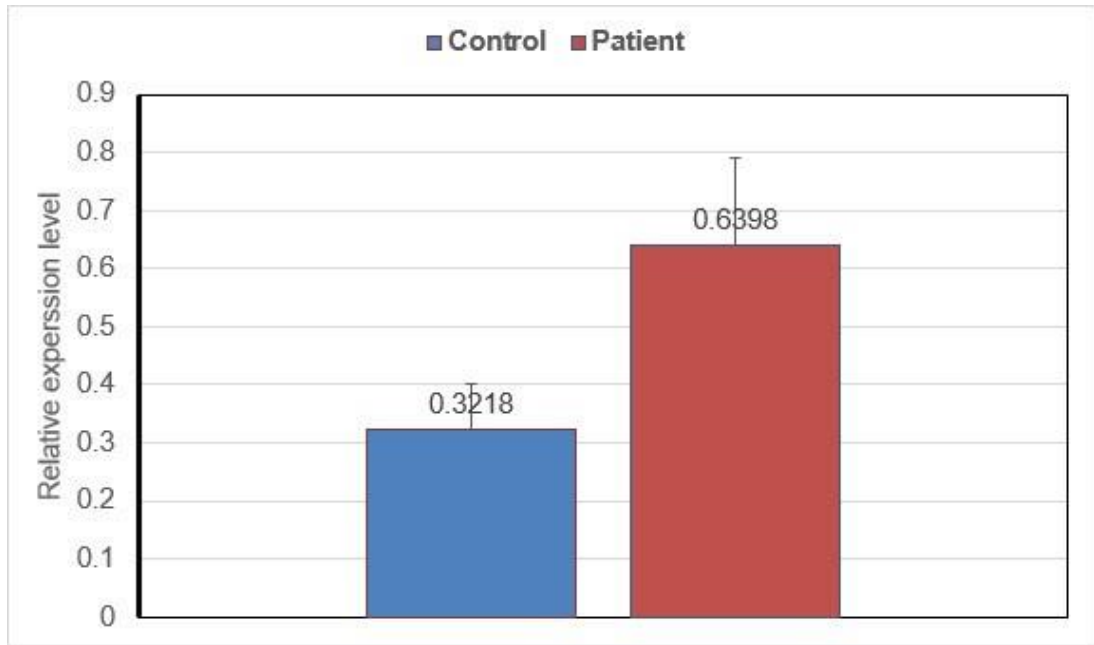

Figure 1. Relative expression of miR-377-3p in healthy and diseased group

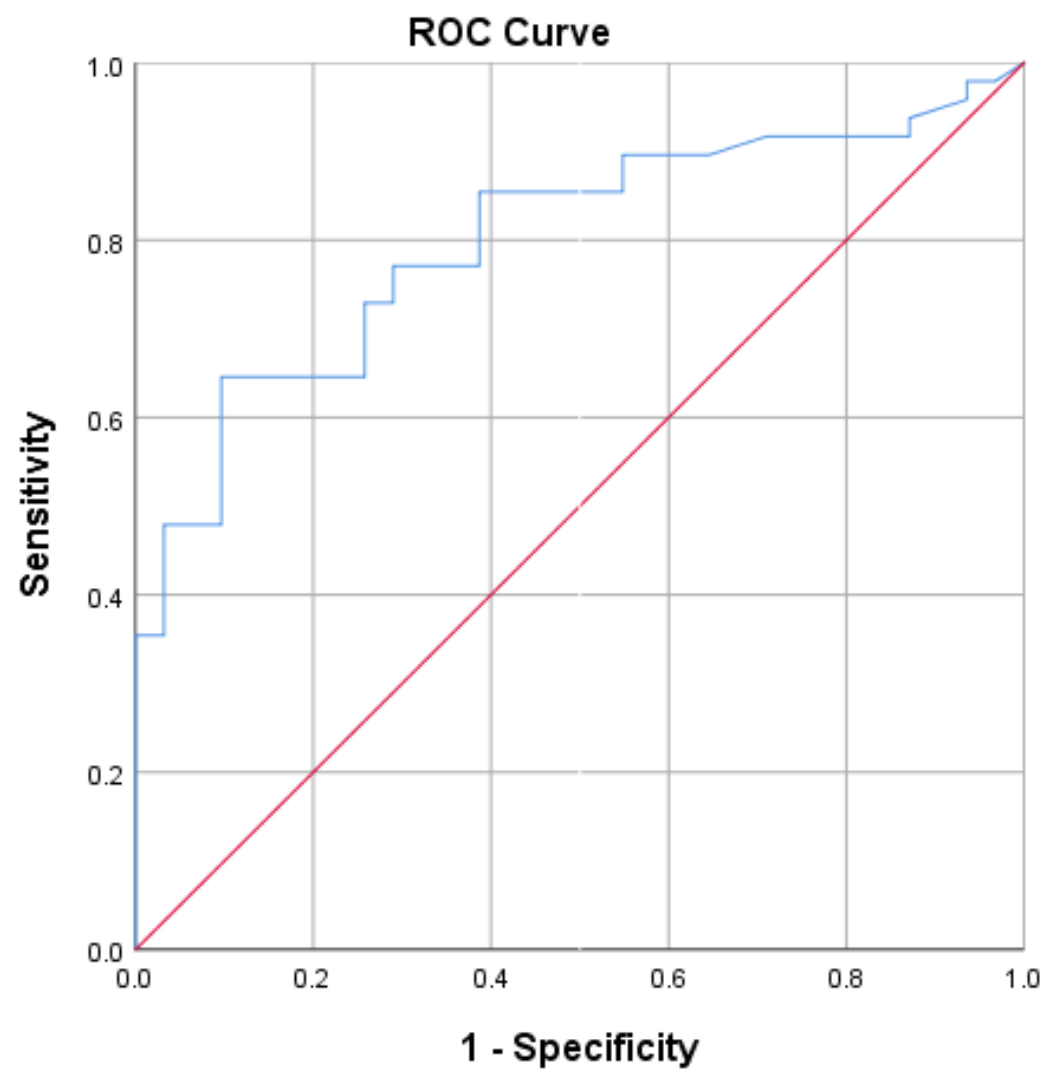

Diagonal segments are produced by ties.

Figure 2. ROC chart, miR-377-3p in healthy and diseased group

\subsection{Discussion}

MS is an autoimmune inflammatory disease of the central nervous system and affects most young people in the community who form the work force, unfortunately in recent years the incidence of MS in the country and especially in the province. Isfahan is on the rise. No one needs to be diagnosed, prevented, or treated promptly in connection with this complex disease. The current treatments for MS have failed to work well and many of them have harmful side effects that are difficult for patients to tolerate. Over the past two decades, with the introduction of microwave RNAs as regulators of gene expression at the post-transcriptional level, another level of complexity in gene expression regulation has emerged. However, microRNAs have been able, with their potential, to give great promise to genetic researchers in the treatment of complex diseases such as cancer and autoimmune diseases such as MS. One of these miRNAs is miR-377-3p, which is located within chromosomal region 32q14, which has been reported in kidney diseases such as diabetes and is in the SOD gene [6]. SOD is a gene located on the chromosomal region 22q21 and the 
protein encoded by this gene is a superoxide dismutase enzyme that is present in humans in three forms: SOD1, SOD2 and SOD3 [7]. Superoxide dismutase 1 is a cytosolic enzyme in humans, a potent antioxidant that protects the body from the damage caused by superoxide, a toxic free radical produced in the mitochondria by converting superoxide radicals to oxygen and hydrogen peroxide. It does. Free radicals produced during cell metabolism can accumulate and damage mitochondrial DNA, nuclei and proteins within the cell. Free radicals are also produced during cellular metabolism such as respiratory bursts and enzymatic reactions [6].

Cells usually have defense mechanisms against ROS-induced damage, and this is a serious issue when ROS production exceeds its antioxidant capacity or the cell's antioxidant system is damaged and this lack the balance between ROS cellular production and the cell's inability to defend itself is called oxidative stress. Oxidative stress causes cell damage and eventually cell death. ROS Attack Glia and Neurons These cells no longer carry mitosis division, so they are highly sensitive to ROS-mediated damage and lead to nerve damage. Inflammation also increases the level of ROS and leads to oxidative stress. One of the most abundant sources of ROS, except the mitochondrial electron transport chain, is the activated microglial respiratory burst system, which induces nitric oxide synthase and releases oxygen and nitrogen radicals leading to myelin degradation and axonal damage. In MS and EAE. Studies in MS patients have shown that free radicals in the CNS of individuals with MS are more active than those of normal subjects and also deficient in antioxidant enzymes. ROS also damages mitochondrial DNA and leads to a decrease in oxidative phosphorylation and thereby loss of neurons [7, 8].

The results of this study showed that miR-377-3p expression was higher in patients than healthy controls. The findings of this study report a study of the association between miR-377-3p expression and the increased risk of MS in Iran, and it is worth noting that a similar study in other countries and provinces of Iran having national and They are ethnic, so far not done.

The results of similar studies by researchers around the world are also different, for example many microRNAs are expressed in batches, and one example of these microRNAs is the miR-379-410 group that contains more than one there are 50 microRNA genes. Studies have shown that miR-379-410 is induced by neuronal depolarization and has an important function in the development of dendrites in cultured hippocampal neurons. There are reports that miR134, a member of this class, is expressed in the dendrites of cultured hippocampal neurons in response to brain-derived neuronal growth factor, or BDNF, with reversible inhibitory effects on LiMK1 kinase mRNA induces dendritic spines. In this case, miR-134 blocks the translation of the mRNA encoding the protein kinase LiMK1 and thus plays a role in the development, maturation and plasticity of the synapse [10, 17].

NMDA-mediated neuronal stimulation also increases miR-219 expression and this microRNA regulates CamKIImediated neuronal excitability. It has been suggested that miR-219 may eventually regulate the expression of NMDA receptors in the synapse and thereby regulate neuronal excitability [15, 16, 18]. Gilgan et al. (2004) investigated the role of oxidative stress in the pathogenesis of MS, and the result of this study is that increased ROS levels increase the damage of cellular components such as lipids, demyelination and axonal degradation. It has a major role in the pathogenesis of MS [7, 19].

Wang et al. (2008) investigated the expression of miR-377 in diabetic patients. In this study, the effect of miR-377 on SOD gene was investigated. The expression of miR-377 was significantly increased in diabetic patients compared to healthy controls and that miR-377 inhibited SOD gene expression and increased the ROS in the body of patients with diabetes. She has diabetes [6].

In a 2009 study by Clare et al., The expression profiles of miRNAs were examined in the blood cells of 20 patients and 19 healthy controls, based on the results: 186-miR, 145-miR, 223-miR, 584-miR, 1275-miR, p5-149-miR, a422miR, p3-142-miR and 664-miR increased expression, while only b20-miR showed decreased expression in MS. Gave. Among the miRNAs studied -186, miR-145 miR showed a significant increase compared to other miRNAs. The 145miR with $90 \%$ sensitivity, 89.5 specificity, and 89.7 accuracy is considered the best marker for healthy and patient differentiation [5].

In this study, we also found that miR-377-3p expression in the MS patient group and healthy group had a significant difference and miR-377-3p expression in the patient group was 1.98 times higher than the healthy group. It was analyzed that by increasing miR-337-3p expression, SOD gene expression was increased and ROS increased, causing damage to cellular components such as lipids and demyelination and axonal degradation. The same damage to cellular components by ROS can increase the degree of disability, or EDSS, in patients with MS. Mitochondria play an important role in ATP production, calcium homeostasis, and apoptosis, where ATP production by mitochondria produces free radicals in the cell, and the superoxide dismutase gene is one of the genes that plays an important role in killing these free radicals. It is produced by mitochondria [10]. 


\section{Conclusion}

The study suggests that increasing miR-377-3p in humans and binding this miR to the superoxide dismutase gene would inhibit SOD gene expression and reduce the amount of superoxide dismutase, which is responsible for lowering the cell's ROS levels and against ROS levels. Increase in cell size. This increase in cell damage and consequently demyelination of more axons and neuronal degradation in MS patients, and this miRNA could potentially accelerate the effect of the SOD gene on MS. Given this significance level, it is desirable that this study be performed in different ethnicities and races and to determine the synchronous relationship between miR-377-3p expression and SOD gene interaction.

\section{Acknowledgements}

The present study was carried out in the framework of the Master Thesis project and thus, the collaboration of all patients who used their blood samples for this study and Dr. Etemadifar's thanks for providing blood samples to patients. Coming.

\section{Declaration of Competing Interest}

The authors declare that they have no known competing financial interests or personal relationships that could have appeared to influence the work reported in this paper.

\section{Ethical Approval}

All procedures performed in studies involving human participants were in accordance with the ethical standards of the institutional and/or national research committee and with the 1964 Helsinki declaration and its later amendments or comparable ethical standards.

\section{References}

[1] Nedjat, S., Montazeri, A., Mohammad, K., Majdzadeh, R., Nabavi, N., \& Nedjat, F. (2006). Multiple sclerosis quality of life comparing to healthy people. Iran J Epidemiol, 1(4), 19-24.

[2] Hauser, S. L., \& Oksenberg, J. R. (2006). The Neurobiology of Multiple Sclerosis: Genes, Inflammation, and Neurodegeneration. Neuron, 52(1), 61-76. doi:10.1016/j.neuron.2006.09.011.

[3] ffrench-Constant, C. (1994). Pathogenesis of multiple sclerosis. The Lancet, 343(8892), 271-275. doi:10.1016/s01406736(94)91118-5.

[4] Nikseresht, A., Izadi, S., \& Rahimi, J. A. (2011). Usage and costs of treatment with beta interferon among patients with multiple sclerosis in Fars province. Hakim Res J. 14(3): 159-164.

[5] Keller, A., Leidinger, P., Lange, J., Borries, A., Schroers, H., Scheffler, M., ... Meese, E. (2009). Multiple Sclerosis: MicroRNA Expression Profiles Accurately Differentiate Patients with Relapsing-Remitting Disease from Healthy Controls. PLoS ONE, 4(10), e7440. doi:10.1371/journal.pone.0007440.

[6] Wang, Q., Wang, Y., Minto, A. W., Wang, J., Shi, Q., Li, X., \& Quigg, R. J. (2008). MicroRNA-377 is up-regulated and can lead to increased fibronectin production in diabetic nephropathy. The FASEB Journal, 22(12), 4126-4135. doi:10.1096/fj.08-112326.

[7] Offen, D., Gilgun-Sherki, Y., \& Melamed, E. (2004). The role of oxidative stress in the pathogenesis of multiple sclerosis: The need for effective antioxidant therapy. Journal of Neurology, 251(3), 261-268. doi:10.1007/s00415-004-0348-9.

[8] Galati, D., Srinivasan, S., Raza, H., Prabu, S. K., Hardy, M., Chandran, K., ... Avadhani, N. G. (2009). Role of nuclear-encoded subunit $\mathrm{Vb}$ in the assembly and stability of cytochrome c oxidase complex: implications in mitochondrial dysfunction and ROS production. Biochemical Journal, 420(3), 439-449. doi:10.1042/bj20090214.

[9] Nathan, C. (2002). Points of control in inflammation. Nature, 420(6917), 846-852. doi:10.1038/nature01320.

[10] Vyshkina, T., Banisor, I., Shugart, Y. Y., Leist, T. P., \& Kalman, B. (2005). Genetic variants of Complex I in multiple sclerosis. Journal of the Neurological Sciences, 228(1), 55-64. doi:10.1016/j.jns.2004.09.027.

[11] Cohen, J. E., Lee, P. R., Chen, S., Li, W., \& Fields, R. D. (2011). MicroRNA regulation of homeostatic synaptic plasticity. Proceedings of the National Academy of Sciences, 108(28), 11650-11655. doi:10.1073/pnas.1017576108.

[12] Dugas, J. C., \& Notterpek, L. (2011). MicroRNAs in Oligodendrocyte and Schwann Cell Differentiation. Developmental Neuroscience, 33(1), 14-20. doi:10.1159/000323919.

[13] Barca-Mayo, O., \& Lu, Q. R. (2012). Fine-Tuning Oligodendrocyte Development by microRNAs. Frontiers in Neuroscience, 6. doi:10.3389/fnins.2012.00013. 
[14] He, X., Yu, Y., Awatramani, R., \& Lu, Q. R. (2011). Unwrapping Myelination by MicroRNAs. The Neuroscientist, 18(1), 4555. doi:10.1177/1073858410392382.

[15] Zhao, X., He, X., Han, X., Yu, Y., Ye, F., Chen, Y., ... Lu, Q. R. (2010). MicroRNA-Mediated Control of Oligodendrocyte Differentiation. Neuron, 65(5), 612-626. doi:10.1016/j.neuron.2010.02.018.

[16] Svaren, J. (2014). MicroRNA and transcriptional crosstalk in myelinating glia. Neurochemistry International, $77,50-57$. doi:10.1016/j.neuint.2014.06.010.

[17] Muddashetty, R., \& Bassell, G. J. (2009). A boost in microRNAs shapes up the neuron. The EMBO Journal, 28(6), 617-618. doi:10.1038/emboj.2009.51.

[18] Li, J.-S., \& Yao, Z.-X. (2012). MicroRNAs: Novel Regulators of Oligodendrocyte Differentiation and Potential Therapeutic Targets in Demyelination-Related Diseases. Molecular Neurobiology, 45(1), 200-212. doi:10.1007/s12035-011-8231-z.

[19] Schratt, G. M., Tuebing, F., Nigh, E. A., Kane, C. G., Sabatini, M. E., Kiebler, M., \& Greenberg, M. E. (2006). A brain-specific microRNA regulates dendritic spine development. Nature, 439(7074), 283-289. doi:10.1038/nature04367. 